\title{
Computation of Bounds for the Positive Eigenvector of a Nonnegative Irreducible Matrix by Monotone Iteration
}

\author{
By W. Bunse and A. Bunse-Gerstner
}

\begin{abstract}
A method for the computation of iterative bounds for the positive eigenvector of a nonnegative irreducible matrix is presented. It is based on the $P$-boundedness of the corresponding fixed point operator. Admissible initial bounds can be obtained by conditional preiteration.
\end{abstract}

1. Introduction. An algorithm for the computation of bounds for the positive eigenvector of a nonnegative irreducible matrix is presented. The method is based on results of Bohl [1] about iterative bounds for the solution of fixed point operator equations. We show that the conditions of a convergence theorem of Bohl can be verified for the corresponding fixed point operator of the eigenvalue equation $M u=\rho(M) u$. The conditions on the starting values can be fulfilled by applying the method of 'conditional iteration' which was introduced by Braess [3] for systems of linear equations. Some connections with the conditions for the cone iteration technique of Sprekels and Voss [7] arise. Some examples demonstrate the performance of our method.

2. $P$-Boundedness of the Fixed Point Operator. Let $M$ be a nonnegative irreducible $N \times N$-matrix. Then the spectral radius $\rho=\rho(M)$ is a simple real eigenvalue with a corresponding positive eigenvector $u, \rho$ is the only eigenvalue of $M$ with a nonnegative eigenvector.

The normalized eigenvector $\|u\|_{1}=\sum_{i=1}^{N} u_{i}=1$ is thus the unique positive solution of the fixed point equation

$$
T x:=\frac{M x}{\|M x\|_{1}}=x .
$$

The computation of iterative bounds for the positive solution of (1) is based on the following convergence theorem of Bohl [1].

Let $Y$ be a nonempty subset of the partially ordered normed vector space $(X, \leqslant,\|\cdots\|)$; let $T$ be an operator on $Y, P$ a linear, $\leqslant$-monotone operator on $X$. $T$ is called $P$-bounded if for all $x, y \in Y$ and $e \in X$ with $x-y \leqslant e$ and $y-x \leqslant e$, we have

$$
T x-T y \leqslant P e \text { and } T y-T x \leqslant P e .
$$

Received March 31, 1981.

1980 Mathematics Subject Classification. Primary 65F15; Secondary 15A48, 15A18. 
If we define the functions

$$
H(v, w):=T\left(\frac{v+w}{2}\right)-P\left(\frac{w-v}{2}\right)
$$

and $L(\alpha, \beta):=(\alpha-\beta, \alpha+\beta)$ for all $(\alpha, \beta) \in Y \times X$, then $P$-boundedness of the operator $T$ is equivalent to monotonicity of $H$ according to $H(v, w) \leqslant H(\tilde{v}, \tilde{w})$ whenever $v \leqslant \tilde{v}$ and $\tilde{w} \leqslant w$ for $(v, w),(\tilde{v}, \tilde{w}) \in L(Y \times X)$. The iteration procedure is defined by

$$
v_{n+1}=H\left(v_{n}, w_{n}\right), \quad w_{n+1}=H\left(w_{n}, v_{n}\right)
$$

under the initial conditions

$$
v_{0} \leqslant H\left(v_{0}, w_{0}\right), \quad v_{0} \leqslant w_{0}, \quad H\left(w_{0}, v_{0}\right) \leqslant w_{0} .
$$

TheOREM 1 (Bohl [1], TheOREM IV, 5.2). Let (A1) or (A2) hold.

(A1): $(X, \leqslant,\|\cdots\|)$ is complete, $Y$ closed,$\rho(P)<1$.

(A2): $T$ and $P$ are completely continuous.

Furthermore let $T$ be P-bounded. Then the sequences $\left(v_{n}\right)_{n \in \mathrm{N}}$ and $\left(w_{n}\right)_{n \in \mathrm{N}}$ from (2), (3) converge to elements $\bar{v}$ and $\bar{w}$, respectively, and $\bar{x}:=\frac{1}{2}(\bar{v}+\bar{w})$ is a solution of $T x=x$. For all $n \in \mathbf{N}$ we have the monotonic error bounds

$$
v_{n} \leqslant v_{n+1} \leqslant \bar{x} \leqslant w_{n+1} \leqslant w_{n} .
$$

Under assumption (Al) $\bar{v}=\bar{w}$ holds.

In the following we shall show the existence of a nonnegative matrix $P$ corresponding to the operator $T x=M x /\|M x\|_{1}$ such that $T$ is $P$-bounded. The condition $\rho(P)<1$ turns out to be equivalent to a condition of Sprekels and Voss [7] and can be accomplished by successive squaring of the matrix $M+I$. This means essentially that the method is restricted to strictly monotone or primitive matrices, a condition, however, which can be overcome by the above-mentioned shift. Starting with known bounds $v_{0}, w_{0}$ for the normalized eigenvector, the initial conditions (3) can be achieved by "conditional" iteration, which was introduced by Braess [3] for systems of linear equations and was also suggested for more general purposes.

Let $K$ be the natural cone of nonnegative elements of $X=\mathbf{R}^{N}$ with canonical partial ordering.

A lower and upper bound for the eigenvector $u \in K$ is given by

$$
v_{0 i}:=\min _{k} \frac{m_{i k}}{\left(e^{T} M\right)_{k}} \leqslant(T x)_{i} \leqslant \max _{k} \frac{m_{i k}}{\left(e^{T} M\right)_{k}}=: w_{0 i}
$$

which is true for all $x \in K$; here we used $e:=(1, \ldots, 1) \in \mathbf{R}^{N}$. (4) can be proved easily by employing the inequality

$$
\min _{i} \frac{p_{i}}{q_{i}} \leqslant \frac{\sum_{j} p_{j}}{\sum_{j} q_{j}} \leqslant \max _{i} \frac{p_{i}}{q_{i}}
$$

which holds for all real numbers $p_{i}$ and positive real numbers $q_{i}$, equality only occurring when all the quotients are equal. Without loss of generality we assume henceforth that $v_{0} \neq 0$.

THEOREM 2. The operator $T x=M x /\|M x\|_{1}$ on the closed set $Y:=\left\{x \in \mathbf{R}^{N} \mid v_{0} \leqslant\right.$ $\left.x \leqslant w_{0}\right\}$ is $P$-bounded for $P:=\left(w_{0}-v_{0}\right) e^{T} M / e^{T} M v_{0}$. 
Proof. The mean value theorem for functions from $\mathbf{R}^{N}$ to $\mathbf{R}$ applied to the component functions $T_{i}$ yields for all $x, y \in \mathbf{R}^{N}$

$$
T x-T y=\left(\begin{array}{c}
\operatorname{grad} T_{1}\left(x+\delta_{1}(y-x)\right) \\
\vdots \\
\operatorname{grad} T_{N}\left(x+\delta_{N}(y-x)\right)
\end{array}\right)(x-y)
$$

for certain $\left.\delta_{i} \in\right] 0,1\left[\right.$. Let $x^{(i)}:=x+\delta_{i}(y-x)$. For the partial derivatives we find

$$
\frac{\partial T_{i}}{\partial x_{j}}\left(x^{(i)}\right)=\frac{m_{i j} e^{T} M x^{(i)}-\left(e^{T} M\right)_{j}\left(M x^{(i)}\right)_{i}}{\left[e^{T} M x^{(i)}\right]^{2}} .
$$

Therefore we have

$$
(T x-T y)_{i} \leqslant \sum_{j=1}^{N}\left|b_{i j}\right||x-y|_{i}
$$

where

$$
b_{i j}:=\frac{m_{i j}}{e^{T} M x^{(i)}}-\frac{\left(e^{T} M\right)_{j}\left(M x^{(i)}\right)_{i}}{\left[e^{T} M x^{(i)}\right]^{2}}
$$

and for $x, y \in Y$

$$
\frac{\left|b_{i j}\right|}{\left(e^{T} M\right)_{j}} e^{T} M x^{(i)}=\left|\frac{m_{i j}}{\left(e^{T} M\right)_{j}}-\frac{\left(M x^{(i)}\right)_{i}}{e^{T} M x^{(i)}}\right| \leqslant w_{0 i}-v_{0 i} .
$$

Because $e^{T} M x^{(i)} \geqslant e^{T} M v_{0}$, we finally have

$$
\left|b_{i j}\right| \leqslant \frac{\left(e^{T} M\right)_{j}}{e^{T} M v_{0}}\left(w_{0 i}-v_{0 i}\right)
$$

The matrix $P$ being dyadic, the condition $\rho(P)<1$ reads

$$
\frac{e^{T} M\left(w_{0}-v_{0}\right)}{e^{T} M v_{0}}<1
$$

This is identical with the condition of Sprekels and Voss' convergence theorem. It was shown there that (5) can always be achieved by taking an appropriate power of $M+I$.

We have shown so far that if the initial values satisfy (3) and if (5) holds, then for the iteration procedure

$$
\begin{aligned}
& v_{n+1}=\frac{M\left(v_{n}+w_{n}\right)}{\left\|M\left(v_{n}+w_{n}\right)\right\|_{1}}-\frac{\left\|M\left(w_{n}-v_{n}\right)\right\|_{1}}{2\left\|M v_{0}\right\|_{1}}\left(w_{0}-v_{0}\right), \\
& w_{n+1}=\frac{M\left(v_{n}+w_{n}\right)}{\left\|M\left(v_{n}+w_{n}\right)\right\|_{1}}+\frac{\left\|M\left(w_{n}-v_{n}\right)\right\|_{1}}{2\left\|M v_{0}\right\|_{1}}\left(w_{0}-v_{0}\right),
\end{aligned}
$$

the statements of Theorem 1 hold.

The proof of Theorem 2 reveals that one can improve convergence by iterating according to

(6) $v_{n+1}=T\left(\frac{v_{n}+w_{n}}{2}\right)-P_{n}\left(\frac{w_{n}-v_{n}}{2}\right), \quad w_{n+1}=T\left(\frac{v_{n}+w_{n}}{2}\right)+P_{n}\left(\frac{w_{n}-v_{n}}{2}\right)$, 
where $P_{n}=\left(w_{0}-v_{0}\right) e^{T} M / e^{T} M v_{n}$. Then we have

$$
\rho\left(P_{n}\right)=\frac{\left\|M\left(w_{0}-v_{0}\right)\right\|_{1}}{\left\|M v_{n}\right\|_{1}} \leqslant \frac{\left\|M\left(w_{0}-v_{0}\right)\right\|_{1}}{\left\|M v_{0}\right\|_{1}}=\rho(P) .
$$

3. Initial Conditions. The initial condition (3) does not automatically hold for the choice of initial vectors $v_{0}, w_{0}$ from (4), but there are methods which generate admissible starting values. Braess [3] has shown how to obtain admissible starting values for monotone iteration schemes for systems of linear equations. We shall briefly sketch how these results carry over to our situation. Other procedures which construct initial values are described by Bohl [1].

The nonnegative cone $K$ of $\mathbf{R}^{N}$ has nonempty interior $\stackrel{\circ}{K}=\left\{x \in \mathbf{R}^{N} \mid x_{i}>0\right.$ for all $i \in\{1, \ldots, N\}\}, x<y$ means $y-x \in \stackrel{\circ}{K}, \sup (x, y)$ and $\inf (x, y)$ are characterized by taking componentwise maxima and minima, respectively.

Starting with the bounds $x_{0}:=v_{0}, y_{0}:=w_{0}$ from (4), the conditional iteration procedure is defined by

$$
\begin{aligned}
& x^{m+1}:=H_{\text {sup }}\left(x^{m}, y^{m}\right):=\sup \left(x^{m}, H\left(x^{m}, y^{m}\right)\right), \\
& y^{m+1}:=H_{\text {inf }}\left(y^{m}, x^{m}\right):=\inf \left(y^{m}, H\left(y^{m}, x^{m}\right)\right) .
\end{aligned}
$$

As in the case of $v_{0 i}=u_{i}=w_{0 i}$ for some component $i$ the iteration is stationary, we may assume without loss of generality that

$$
x_{0}<u<y_{0} .
$$

Then $\rho(P)<1$ implies the existence of a number $m_{0}$ such that

$$
x^{0}<x^{m_{0}} \leqslant y^{m_{0}}<y^{0} .
$$

This can be seen as follows:

The definition of $H$ and of the conditional iteration procedure implies $y^{m}-x^{m} \leqslant$ $P^{m}\left(y^{0}-x^{0}\right)$, from which we conclude that

$$
\lim _{m \rightarrow \infty}\left(y^{m}-x^{m}\right)=0 .
$$

By induction we obtain

$$
x^{m} \leqslant x^{m+1} \leqslant u \leqslant y^{m+1} \leqslant y^{m},
$$

making use of the $P$-boundedness of $T$. Together with (7) and (9), the stated result (8) follows.

(8) in turn now implies that $x^{m_{0}-1}, y^{m_{0}-1}$ are admissible starting values for the ordinary procedure (2) and (3). This can be seen by noting that whenever $x_{i}^{m+1}=$ $H\left(x^{m}, y^{m}\right)_{i}$ for an $m \in \mathbf{N}$ and a component $i$, then this also holds for all $n \geqslant m$ $\left(\right.$ similarly for $\left.y^{m}\right)$.

The condition $\rho(P)<1$ is also necessary for conditional iteration to lead to situation (8). Assume $\rho(P) \geqslant 1$; let $x \geqslant 0$ be an eigenvector of $P$ corresponding to the eigenvalue $\rho(P)$. By assumption (8) the ordinary iteration takes over, so by Theorem 1 there exist

$$
\begin{gathered}
\bar{x}:=\lim _{m \rightarrow \infty} H_{\text {sup }}\left(x^{m}, y^{m}\right), \quad \bar{y}:=\lim _{m \rightarrow \infty} H_{\text {inf }}\left(x^{m}, y^{m}\right) \quad \text { and } \\
\bar{x}=T\left(\frac{\bar{x}+\bar{y}}{2}\right)-P\left(\frac{\bar{y}-\bar{x}}{2}\right) .
\end{gathered}
$$


Because $x^{0}<\bar{x}$ and $\bar{y}<y^{0}$, there exists $\varepsilon \in \mathbf{R}_{+}$such that

$$
x^{0} \leqslant \bar{x}-\varepsilon x \text { and } y^{0} \geqslant \bar{y}+\varepsilon x .
$$

Monotonicity of $H$ yields

$$
\begin{aligned}
H\left(x^{0}, y^{0}\right) & \leqslant H(\bar{x}-\varepsilon x, \bar{y}+\varepsilon x)=T\left(\frac{\bar{x}+\bar{y}}{2}\right)-P\left(\frac{\bar{y}-\bar{x}}{2}\right)-P(\varepsilon x) \\
& \leqslant \bar{x}-\varepsilon x .
\end{aligned}
$$

From (10) and (11) we have

$$
x^{1}=\sup \left(x^{0}, H\left(x^{0}, y^{0}\right)\right) \leqslant \bar{x}-\varepsilon x
$$

and similarly

$$
y^{1}=\inf \left(y^{0}, H\left(y^{0}, x^{0}\right)\right) \geqslant \bar{y}+\varepsilon x .
$$

Then by induction

$$
x^{m} \leqslant \bar{x}-\varepsilon x \quad \text { for all } m \in \mathbf{N} .
$$

The order relation being closed, this leads to a contradiction.

4. Numerical Computations. All of the several conditions for the proposed method to work can be fulfilled for an arbitrary nonnegative irreducible matrix $M$ by considering appropriate powers of $M+I$. The shift is necessary only for imprimitive matrices. We recall that these conditions included a nontrivial lower bound for the eigenvector, the condition on the spectral radius of $P$, and the initial conditions (3). As $\rho(P)$ governs the rate of convergence essentially it is usually advisable to square $M+I$ beyond the point where $\rho(P)$ is first less than 1. To avoid arithmetic overflow or underflow one then has to eventually normalize the matrix.

The following examples were computed with the improved method, i.e., using $P_{n}$ defined in (6) instead of $P$. As a measure of accuracy we required the relative error of the eigenvector $\left\|w_{n}-v_{n}\right\|_{1} /\left\|v_{n}\right\|_{1}$ and the relative error of the eigenvalue $\left(\left\|M w_{n}\right\|_{1}-\left\|M v_{n}\right\|_{1}\right) /\left\|M v_{n}\right\|_{1}$ to be less than $10^{-8}$. The computations were performed on the TR 440 of the University of Bielefeld.

Example 1. The $N \times N$-matrix

$$
M=\left(\left(m_{i j}\right)\right), \quad m_{i j}:=N+1-\max (i, j),
$$

has spectral radius $\rho(M)=\frac{1}{2}[1-\cos (\pi /(2 N+1))]^{-1}$.

(a) For $N=12$ it was computed by Yamamoto [8] and Hall and Spanier [6] with their modifications of the power method. For $M$ itself we have $\rho(P)=0.6304$, $v_{0} \neq 0$. After three conditional iteration steps condition (3) was satisfied and after a total number of 25 iterations the desired accuracy was reached. The corresponding $P$ for $(M+I)^{2}$ has a spectral radius of 0.0632 , and only six iterations lead to the same accuracy, taking about one third of the computational time.

Further squaring gives a spectral radius of $\rho(P)=0.001$. Observe that $\rho(P)$ is also an a priori relative error of the eigenvalue $\rho(M)$. For $(M+I)^{4}$ only two iterations were necessary to obtain the relative error of $10^{-8}$. The additional squaring, however, takes about as much time as the saved four iterations so that there was no further saving in computational time.

(b) Whether additional squaring results in saving computational time also depends on the size of the matrix. This is illustrated by the following table. After each $N$ we 
list the number of squarings of the matrix, the spectral radius $\rho(P)$, the total number of iterations (number of conditional iterations), and the computation time in seconds.

\section{TABLE 1}

\begin{tabular}{ccccc|ccccc} 
& \# squ. & $\rho(P)$ & \# it. & sec & & \# squ. & $\rho(P)$ & \#it. & sec \\
$N=3$ & 0 & 0.4400 & $18(1)$ & 0.45 & $N=6$ & 0 & 0.5778 & $23(2)$ & 0.72 \\
& 1 & 0.2584 & 12 & 0.32 & & 1 & 0.1174 & 8 & 0.44 \\
& 2 & 0.0300 & 5 & 0.26 & & 2 & 0.0031 & 3 & 0.32 \\
\hline$N=12$ & 0 & 0.6304 & $25(3)$ & 1.95 & $N=24$ & 0 & 0.6572 & $26(5)$ & 3.53 \\
& 1 & 0.0632 & 6 & 0.64 & & 1 & 0.0513 & 6 & 1.82 \\
& 2 & 0.0009 & 2 & 0.59 & & 2 & 0.0006 & 2 & 1.88 \\
\hline$N=48$ & 0 & 0.6707 & $27(6)$ & 11.09 & $N=96$ & 0 & 0.6774 & $27(7)$ & 38.60 \\
& 1 & 0.0485 & 6 & 7.09 & & 1 & 0.0478 & 6 & 35.12 \\
& 2 & 0.0006 & 2 & 9.04 & & 2 & 0.0006 & 2 & 55.90
\end{tabular}

Example 2. For the computation of the spectral radius and the positive eigenfunction of the integral operator

$$
K f(s):=\int_{-1}^{1} \frac{1}{\sqrt{(s-t)^{2}+1}} f(t) d t
$$

a product Simpson's method was used, i.e., replacing the kernel function $k(s, t)$ in the variable $t$ and the unknown eigenfunction by piecewise quadratic polynomials and then collocating. This is equivalent to replacing the kernel function by a biquadratic polynomial.

Using $N+1$ equidistant nodes in each direction, this amounts to solving the $(N+1) \times(N+1)$-matrix eigenvalue problem

$$
\tilde{K} f_{N+1}:=K_{N+1} W_{N+1} f_{N+1}=\lambda f_{N+1},
$$

where $K_{N+1}$ consists of values $k\left(s_{i}, t_{j}\right)$ at the nodes, $f_{N+1}=\left(f\left(s_{0}\right), \ldots, f\left(s_{N}\right)\right)^{T}$, and the weight matrix is given by

$$
W_{N+1}=\frac{1}{15 N}\left[\begin{array}{rrrrrrrrr}
4 & 2 & -1 & & & & & & \\
2 & 16 & 2 & & & & 0 & & \\
-1 & 2 & 8 & 2 & -1 & & & & \\
& & 2 & 16 & 2 & & & & \\
& & -1 & 2 & 8 & & & & \\
& & & & & \ddots & & & \\
& 0 & & & & & 8 & 2 & -1 \\
& & & & & & 2 & 16 & 2 \\
& & & & & & -1 & 2 & 4
\end{array}\right]
$$

(cf. Hämmerlin [5] and Boland and Duris [2]). Burgmeier and Scott [4] have shown that for the integral operator $K 1.65711885 \leqslant \rho(K) \leqslant 1.65711891$ holds. In the following table we list the bounds for the spectral radius of the discrete approximation operators $\tilde{K}$ and the characteristics of the iteration as in Example 1. 
TABLE 2

$\begin{array}{ccrc}N=10(1.657 & 12859 \leqslant \rho(\tilde{K}) \leqslant 1.657 & 12860) \\ \# \text { squ } & \rho(P) & \# \text { it. } & \text { sec } \\ 0 & 0.6051 & 25 & 1.25 \\ 3 & 0.2157 & 11 & 0.96 \\ 4 & 0.0410 & 5 & 0.81\end{array}$

\begin{tabular}{cccc}
$N=$ & \multicolumn{4}{c}{$(1.657119$} & 43 & \multicolumn{2}{c}{$\rho(\tilde{K}) \leqslant 1.65711944)$} \\
0 & 0.5963 & 24 & 2.75 \\
3 & 0.3561 & $17(1)$ & 3.29 \\
4 & 0.0681 & 6 & 2.68
\end{tabular}

$N=40(1.65711889 \leqslant \rho(\tilde{K}) \leqslant 1.65711891)$

$\begin{array}{llcr}0 & 0.5913 & 24 & 7.84 \\ 4 & 0.1223 & 8(1) & 12.27 \\ 5 & 0.0603 & 6(1) & 14.20\end{array}$

\begin{tabular}{cccc}
$N=$ & \multicolumn{4}{c}{$(1.657118$} & 86 & \multicolumn{2}{c}{$\rho(\tilde{K}) \leqslant 1.65711887)$} \\
0 & 0.5891 & 24 & 25.33 \\
4 & 0.2307 & $12(2)$ & 73.49 \\
6 & 0.0917 & $7(1)$ & 99.28
\end{tabular}

From the computed examples we conclude that additional squaring is worthwhile only for small $N$.

Fakultät für Mathematik

Universität Bielefeld

Postfach 8640

D4800 Bielefeld 1, Federal Republic of Germany

1. E. BoHL, Monotonie: Lösharkeit und Numerik bei Operatorgleichungen, Springer-Verlag, Berlin, Heidelberg and New York, 1974.

2. W. R. Boland \& C. S. DuRis, "Product type quadrature rules," BIT, v. 11, 1971, pp. 139-158.

3. D. BRAESS, "Die Konstruktion monotoner Iterationsfolgen zur Lösungseinschließung bei linearen Gleichungssystemen," Arch. Rational Mech. Anal., v. 9, 1962, pp. 97-106.

4. J. W. BURgmeier \& M. R. SCOTt, "A method for obtaining bounds on eigenvalues and eigenfunctions by solving non-homogeneous integral equations," Computing, v. 10, 1972, pp. 9-22.

5. G. HÄMmERLIN, "Ein Ersatzkernverfahren zur numerischen Behandlung von Integralgleichungen 2. Art," Z. Angew. Math. Mech., v. 42, 1962, pp. 439-463.

6. C. A. Hall \& J. SPanier, "Nested bounds for the spectral radius," SIAM J. Numer. Anal., v. 5, 1968, pp. 113-125.

7. J. SPREKELS \& H. Voss, “Ein Verfahren zur iterativen Einschließung des positiven Eigenvektors einer irreduziblen, nichtnegativen Matrix,” Computing, v. 20, 1978, pp. 27-34.

8. T. Yамамото, "A computational method for the dominant root of a non-negative irreducible matrix," Numer. Math., v. 8, 1966, pp. 324-333. 